\title{
Wie viel Gesundheit kosten darf
}

\section{Rahel Gutmann}

Junior-Redaktorin der Schweizerischen Ärztezeitung

\author{
Zu ihrem 125. Jubiläum zeigt die Roche Werke des britischen Künstlers Paddy Hart- \\ ley. Der Brite setzt sich kreativ mit Medizin und Forschung auseinander und geht \\ der Frage nach, wie wir sie und sie uns formen. Ein Rundgang durch die Ausstel- \\ lung «The cost of life» im Basler Museum Tinguely.
}

Helle Kunstwerke auf weissen Präsentiersäulen und -blöcken. Sehr übersichtlich, nahezu clean kommt der Ausstellungsraum direkt neben dem Eingang im $\mathrm{Mu}-$ seum Tinguely daher. Findet sich in der Raumgestaltung eine erste Anspielung auf die Medizin und ihre Erkennungsfarbe Weiss? Oder soll sie vielleicht darauf verweisen, dass der Bildhauer und Keramiker Paddy Hartley für die Ausstellung vorwiegend mit heller Keramik gearbeitet hat? Vielleicht ja beides.

Hartley beschäftigt sich schon lange mit Fragen zu den Grenzbereichen in Medizin und Forschung und beleuchtet dabei vor allem die Sicht der Patientinnen und Patienten. Schliesslich sind sie diejenigen, deren Leben mit Hilfe von Medikamenten, medizinischen Eingriffen und wissenschaftlichen Berechnungen verbessert oder doch zumindest verlängert werden soll. Diese thematische Ausrichtung erklärt auch, weshalb die Roche genau ihn für die Sonderausstellung «The cost of life» anfragte.

\section{Anfänge liegen bei der Gesichtschirurgie}

Passend zum langjährigen Bestehen der Roche widmet sich der Künstler medizinischen Entwicklungen in Ge- schichte und Gegenwart: Vom Entstehen der rekonstruktiven Gesichtschirurgie während des 1. Weltkriegs, bis zu ihrem Einsatz in der Schönheitschirurgie.

Eine Entwicklung, die den Bezug zur Gegenwart herstellt und sich im Werk Looking Glass zeigt: Die Betrachterin bzw. der Betrachter blickt auf eine schwarze Spiegelfläche, umrandet von einem ins Hässliche gesteigerten, wulstigen Rahmen im barocken Stil - glasiert und rosa. Die schwarze Fläche steht für den Bildschirm eines Smartphones und damit für die Oberflächlichkeit und das Zurschaustellen im digitalen Zeitalter.

\section{Sinn und Unsinn in der Medizin}

Richtet sich die Medizin folglich primär nach dem Bedarf der Patientinnen und Patienten und formt sich anhand ihrer Wünsche? Hartley könnte dahingehend verstanden werden. Gerade auch wenn man das Werk You Can Have any Color, as Long as it's Blue betrachtet, in dem die In-vitro-Fertilisation angesprochen wird. Ausgestellt ist ein in eine Laborzentrifuge integriertes Roulette-Rad, in dem die Kugel nur auf der Option «blaue Augen» landen kann. Denn Hartley ist überzeugt: die

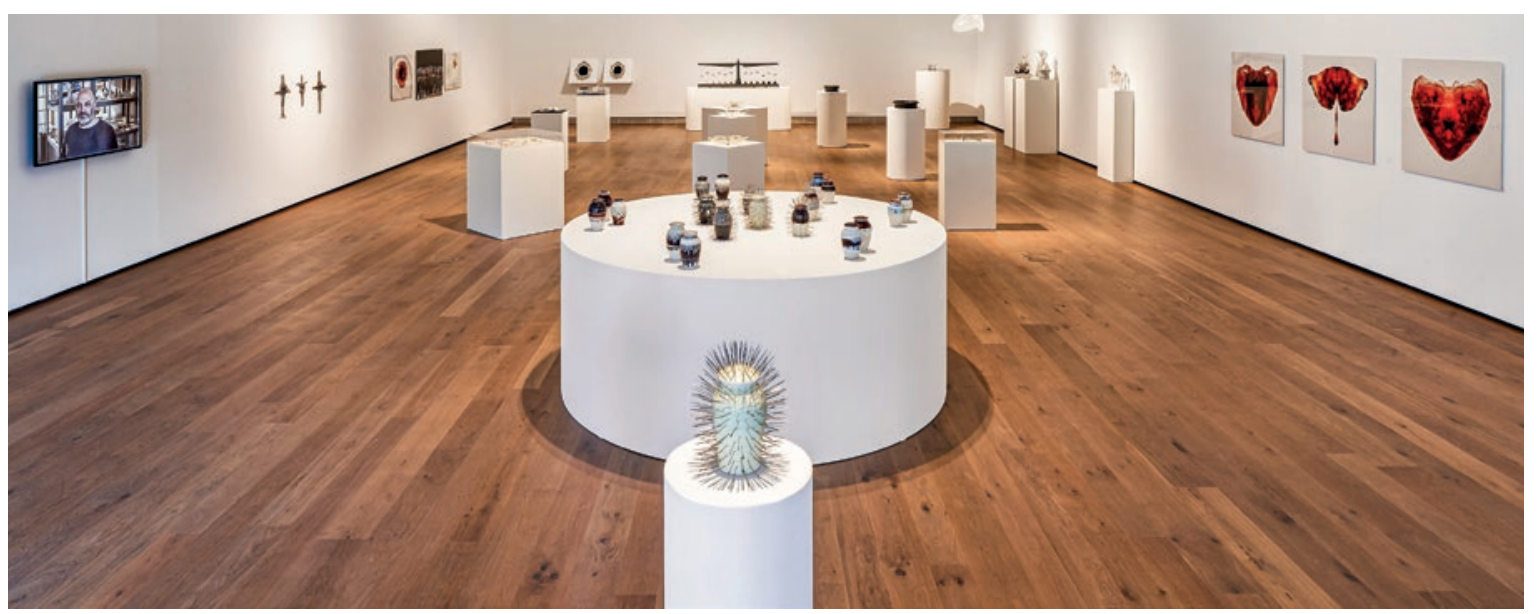

Der Ausstellungsraum im Museum Tinguely (C) 2021, Museum Tinguely/Daniel Spehr). 
wachsenden Möglichkeiten im Bereich der Fortpflanzungsmedizin werden dazu führen, dass werdende Eltern zusehends ein verbessertes Abbild ihrer selbst kreieren wollen. Demnach schwingt auch hier die Frage mit, ob und wie wichtig das Aussehen sein sollte und wo die Grenze überschritten wird zwischen sinnvollen medizinischen Anwendungen und Schönheitskult.

Doch richtet die Ausstellung den Blick nicht nur kritisch auf die Verbraucherin und den Verbraucher, sondern ebenso auf die medizinische Wissenschaft und Forschung. Eine grosse Waage an der Rückseite des Ausstellungsraums, die Infinity Balance, setzt sich mit der Bedeutung wissenschaftlicher Voraussagen auseinander. Sie nimmt Bezug auf ein Symposium mit dem Title «The Challenge of Life», das Roche zu ihrem 75-Jahr-Jubiläum veranstaltet hatte und das sich um Fragen der damals noch jungen biomedizinischen Forschung drehte. Im Hintergrund stand die prognostizierte Überbevölkerung der Erde, die dann durch die Entwicklung und Verbreitung von Kontrazeptiva doch nicht eintrat. Dafür entstanden neue, unvorhergesehene Probleme wie die Überalterung der Bevölkerung. Manche Probleme verschwinden, dafür kommen andere hinzu, darauf verweist die Unendlichkeitswaage.

\section{Von der Pandemie geprägt}

Viele Ausstellungsstücke sind geprägt von ihrer Entstehungszeit in der Covid-19-Pandemie. So stehen in der

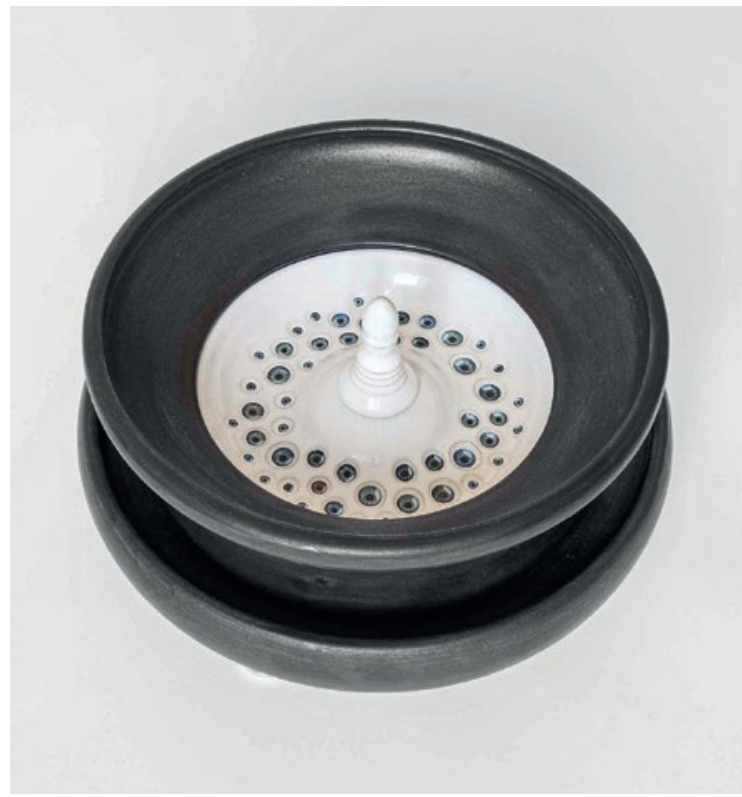

Paddy Hartley, You Can Have any Color, as Long as it's Blue, 2021 () Basile Bornand).

Mitte des Raumes mit Kanülen gespickte Keramikurnen, und mit gebührendem Abstand stehen darum herum kleinere Urnen ohne Kanülen. Damit wird symbolisiert, dass viele Angehörige ihre sterbenden Verwandten wegen der Besuchsverbote in Gesundheitseinrichtungen nicht mehr sehen und Abschied nehmen konnten. Die Kanülen verweisen zusätzlich darauf, wie der Tod mit Hilfe von medzinischen Mitteln abgewehrt werden soll.

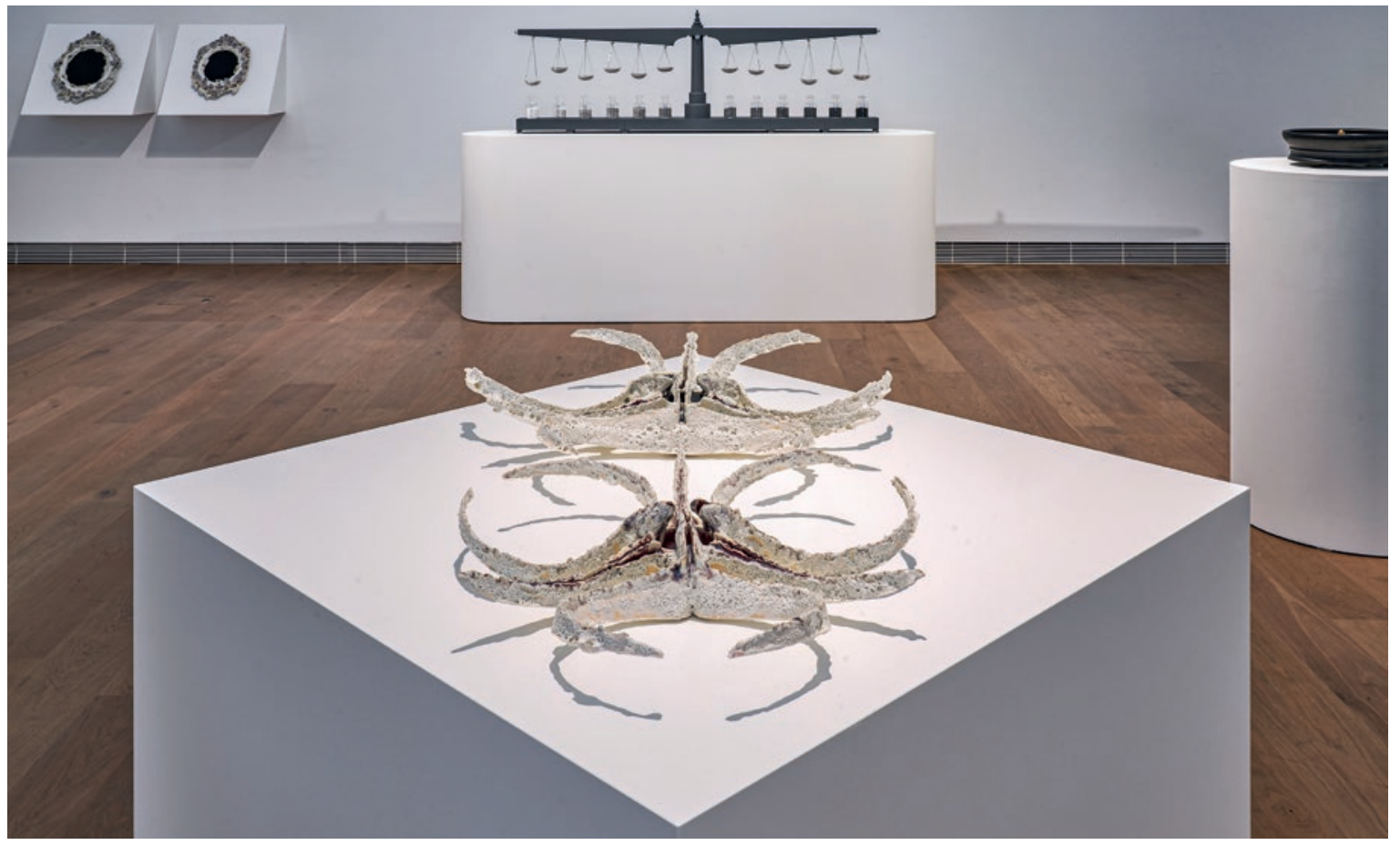

Installationsansicht: Links ist das Looking Glass zu sehen, in der Mitte hinten die Infinity Balance und im Vordergrund die III Communication Dishes (@ 2021, Museum Tinguely/Daniel Spehr). 


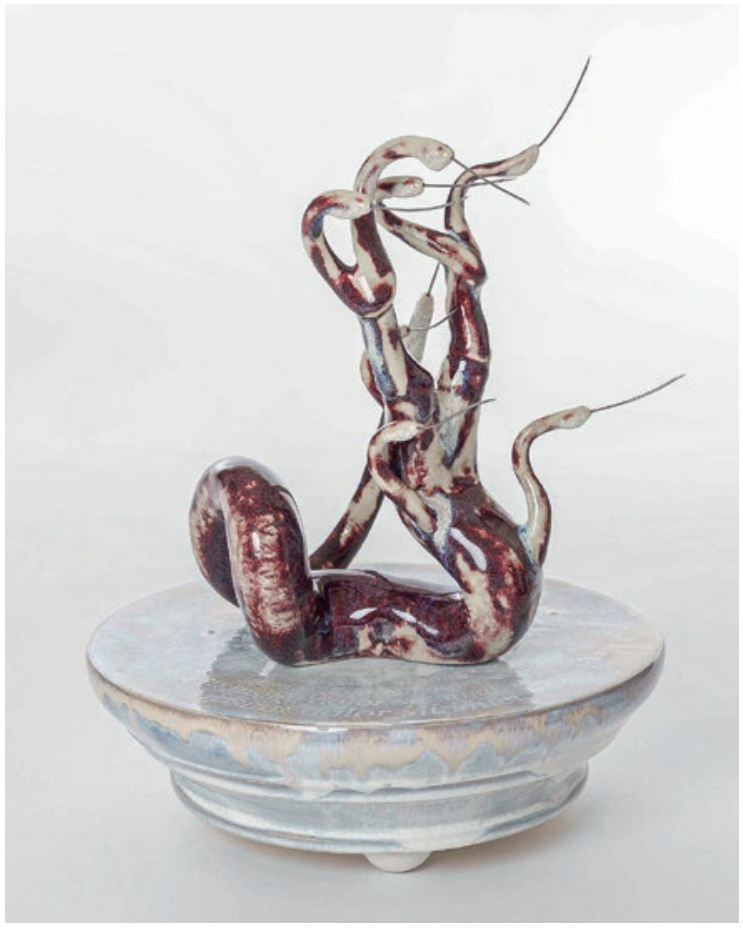

Paddy Hartley, HypoTrypanoPharmAlethephobia or: The Frustration of the Virologist, 2021 (ㄷ) Basile Bornand).

Die vieldiskutierte Impfskepsis diente als Inspiration für das Werk The Frustration of the Virologist, das aus hydra-artigen Schlangenfiguren besteht. Hinzu kommen die III Communication Dishes aus filigranen Keramikformen, die an Überreste von überdimensionalen Krebsen erinnern, tatsächlich aber Satellitenschüsseln ähneln sollen. Sie haben eine dreigeteilte Struktur, die das Wifi-Symbol sowie das Gefahrensymbol für Biogefahren aufgreift. Damit wird das Thema der Falschin-

\section{Ausstellung «The Cost of Life»}

Die Ausstellung "The Cost of Life» ist noch bis zum 23. Januar im Museum Tinguely zu sehen.

Sie wird ergänzt durch eine Sonderausstellung im Pharmazie-

rahel.gutmann[at]emh.ch formation im Internet thematisiert und die feinen, aber gefährlichen Netzwerke, die dadurch entstehen.

\section{Zur Nähe von Glaube und Medizin}

Eine weitere Symbolik sticht im Ausstellungsraum heraus: Rosenkränze aus Pillen, eine Dornenkrone aus Pipetten sowie kreuzförmig zusammengesetzte Hüftprothesen verweisen auf die Ähnlichkeit zwischen religiösen Überzeugungen und der Überhöhung medizinischer Errungenschaften. In beide setzen die Menschen grosse Hoffnung, und beide haben das Potenzial, zu helfen oder zu schaden. Gleichzeitig zeigen die für die Rosenkränze verwendeten Pillen, wie leicht es ist, über das Internet an Medikamente wie Viagra oder Prozac zu kommen. Als Experiment hatte Hartley sie selbst bestellt.

Beim Gang durch die Ausstellung erscheinen die Werke mit ihren rosa oder weiss glasierten Keramikoberflächen harmlos, vereinen jedoch eine grosse thematische Bandbreite und greifen schwierige Fragen auf. Bei vielen Stücken scheint die persönliche Haltung des Künstlers und damit eine Kritik an gewissen Überzeugungen und Handlungsweisen durch. So wird man als Besucherin und Besucher herausgefordert, nicht nur zum Kunstwerk, sondern auch zur Haltung des Künstlers Stellung zu beziehen - sei dies aus Patientensicht, als Forschende oder als Ärztin und Arzt.

\section{Jubiläumsjahr geht zu Ende}

Das Jahr 2021 markierte das 125-Jahr-Jubiläum des Pharmakonzerns Roche, der am 1. Oktober 1886 unter dem Namen F. Hoffmann-La Roche \& Co. gegründet worden war. Seither hat sich Roche zu einem internationalen Unternehmen mit Standorten in über 100 Ländern entwickelt. Der Hauptsitz ist bis heute in Basel geblieben. Um die Verbundenheit des Unternehmens mit der Stadt auszudrücken, hatte Roche der Stadt zum 100-JahrJubiläum das Museum Tinguely gestiftet. Zum Anlass des diesjährigen Jubiläums hat sie den Basler Zoo mit einer Schenkung bedacht und unter dem Motto "Celebrate life» verschiedene kulturelle und musikalische Veranstaltungen durchgeführt. 